\title{
Impacto de las revistas españolas de Biblioteconomía y Documentación y repercusión de las autocitas en su índice $h$
}

\author{
José Antonio Salvador-Oliván* \\ Gonzalo Marco-Cuenca* \\ Rosario Arquero-Avilés**
}

Artículo recibido:

17 de octubre de 2017

Artículo aceptado:

10. de agosto de 2018

Artículo de investigación
Resumen

El número de citas que recibe un artículo es un indicador de su impacto en la comunidad científica. El objetivo de este trabajo es analizar el impacto de los artículos más citados que han sido publicados en las dos revistas españolas de Biblioteconomía y Documentación incluidas en el Journal Citation Reports (JCR), así como determinar la influencia de las autocitas en su índice h. Los resultados muestran que los artículos son citados principalmente por autores y revistas iberoamericanas, con un impacto internacional muy bajo debido, prácticamente en su totalidad, a la presencia

* Departamento de Ciencias de la Documentación e Historia de la Ciencia Universidad de Zaragoza, España._ jaso@unizar.es gmarco@unizar.es

** Departamento de Biblioteconomía y Documentación Universidad Complutense de Madrid, España._ carquero@ucm.es

INVESTIGACIÓN BIBLIOTECOLÓGICA, vol. 32, núm. 77, octubre/diciembre, 2018, México, ISSN: 2448-8321 pp. 13-30 
de artículos escritos en inglés. Las revistas más citantes son las mismas para ambos casos, la revista mexicana Investigación Bibliotecológica, las revistas españolas El Profesional de la Información (EPI) y Revista Española de Documentación Cientifica (REDOC), y una revista internacional (Scientometrics). La tasa de autocitación conjunta de revistas y autores es muy alta (40\%); la eliminación de las autocitas de revista afecta al índice h y proporciona una medida más objetiva y real del impacto.

Palabras clave: Revistas Españolas; Biblioteconomía y Documentación; Web of Science; Impacto; Visibilidad; Autocitas; Índice h

Impact of the Spanish Library and Information Science journals and impact of self-citation on their $\mathrm{H}$-index José Antonio Salvador-Oliván, Gonzalo Marco-Cuenca and Rosario Arquero-Avilés

\section{Abstract}

The number of citations article paper receives serves as an indicator of its impact in the scientific community. This paper performs an analysis of the impact of the most frequently cited papers published in two Spanish Library and Information Science journals included in Journal Citation Reports (JCR) and determines the influence of self-citations on their $\mathrm{H}$ index. Results show that the papers are cited largely by Latin-American authors and journals with little international impact, whose scant impact, in fact, is almost entirely due to papers written in English included in the journal. The most frequently citing journals for both papers are the Mexican journal Investigación Bibliotecológica, the Spanish journals, El Profesional de la Información (EPI), and Revista Española de Documentación Cientifica (REDOC), and the international journal Scientometrics. The self-citation rate of these journals and authors is very high (40\%); and the elimination of self-citations impacts the H-index while yielding a more objective, realistic measures of impact.

Keywords: Spanish Journals; Library and Information Science; Web of Science; Impact; Visibility; Self-citation; LIS Spanish Journals; H-index 


\section{INTRODUCCIÓN Y OBJETIVOS}

— $\mathrm{n}$ la cultura científica actual predomina el reconocimiento profesional ¿que supone la publicación en revistas de prestigio (Schekman, 2013). Este prestigio se basa en el impacto, medido a través del número de citas que reciben las publicaciones y/o revistas. La principal fuente de datos de la que se obtienen las citas y los indicadores derivados de ellas es la base de datos Web of Science (WoS), que contiene las revistas más importantes (mainstream) de las distintas áreas científicas; la publicación de artículos en estas revistas es un requisito en el procedimiento de evaluación de la investigación de profesores universitarios e investigadores. Sin embargo, se han señalado algunas limitaciones como su sesgo en la cobertura idiomática (principalmente revistas en lengua inglesa) y otras que afectan a los indicadores de impacto.

La indización de una revista en WoS se considera un símbolo de prestigio y su clasificación en cuartiles, según el número de citas recibidas, revela su importancia e impacto en la comunidad científica internacional. Habitualmente, se utilizan como indicadores de impacto el factor de impacto (FI) y el FI de 5 años para las revistas del Journal Citation Reports (JCR), y el Scimago Journal Rank (SJR) para las revistas indizadas en Scopus. Aunque son muchas las razones y los factores que contribuyen a que un artículo sea citado, el número de citas demuestra que un artículo ha sido empleado en la literatura y, por tanto, estos indicadores se han utilizado como una aproximación del nivel de influencia e internacionalización de las revistas.

Entre las muchas críticas que han recibido los indicadores de impacto (Bordons, Fernández y Gómez, 2002; Vanclay, 2012) se encuentra la influencia de las autocitas. El término autocita se ha utilizado para indicar que el artículo citante y el citado tienen uno o más autores en común (autocita de autoría) o cuando los artículos citantes están publicados en la misma revista que los artículos citados (autoría de revista) (Egghe y Rousseau, 1990).

Las autocitas forman parte natural de la comunicación científica y son, en sí mismas, útiles, relevantes y funcionales en el proceso de publicación y transferencia del conocimiento (Opthof, 2013), además de configurarse como un fenómeno que es necesario medir (Gorbea-Portal y Suárez-Balseiro, 2007). No obstante, también pueden ser inapropiadas y utilizarse como estrategia de promoción, tanto de los propios autores para mejorar su visibilidad e impacto (Hyland, 2003) como de las revistas cuando los editores sugieren a los autores que citen artículos publicados en sus revistas -coercive journal self citation- (Ioannidis, 2015; Wilhite y Fong, 2012; Foley y Della Sala, 2010), consiguiendo con estas malas prácticas sobredimensionar su impacto percibido, un fenómeno cada vez más frecuente en todos los ámbitos 
científicos (Chorus y Waltman, 2016). Por estas razones, son varios los autores que se decantan por su eliminación, ya que no reflejan el impacto real de una publicación (Aksnes, 2003; Schreiber, 2007) y suponen una perversión de los propios índices (Espinet Rubio, 2006); incluso algunos autores proponen la penalización de las autocitas de revista (De Granda Orive, Alonso Arroyo y Aleixandre Benavent, 2014).

Son varios los estudios que han analizado los efectos de las autocitas en el factor de impacto de las revistas y con resultados contradictorios. En algunos se ha hallado que las autocitas aumentan el factor de impacto de las revistas (Krauss, 2007; Frandsen, 2007), mientras que otros sugieren que su efecto es insignificante (Finardi, 2013; Campanario, 2010), incluido un estudio propio del antiguo Thomson Reuters en el que se concluye que el rango de las revistas en el JCR se ve poco alterado, sobre todo en aquellas revistas con mayor factor de impacto (McVeigh, 2002).

A lo largo de los años se han ido introduciendo modificaciones en los indicadores con el propósito de eliminar o mitigar el efecto de las autocitas y, de esta manera, reflejar con mayor precisión su impacto en la comunidad científica. Así, el factor de impacto, el Eigenfactor Score y el Article Influence Score excluyen las autocitas, mientras que el SIR limita el número de autocitas en su fórmula.

También se han desarrollado otros indicadores como el índice h, que combina tanto la producción científica como el impacto. Propuesto inicialmente por Hirsch para cuantificar la producción científica de los investigadores (Hirsch, 2005), comenzó a utilizarse también para evaluar las revistas (Braun, Glänzel y Schubert, 2006), siendo un indicador que aporta nueva información y que, por tanto, puede complementar a los indicadores de impacto (Salvador Oliván y Agustín Lacruz, 2015).

Se ha cuestionado la robustez del índice h ya que, al igual que cualquier indicador que se base en el número de citas, es susceptible de ser alterado por la autocitación; a pesar de ello, es un índice que se publica en las distintas bases de datos sin ser corregido por las autocitas. La mayoría de las publicaciones que han estudiado esta relación se centra en el índice h de los autores, y aunque Hirsch argumentó que el efecto de las autocitas es relativamente pequeño (Hirsch, 2005), son varios los estudios que han demostrado que puede ser importante (Bartneck y Kokkelmans, 2011), sobre todo en los autores con índices h más bajos (Gianoli y Molina-Montenegro, 2009) y se recomienda eliminarlas de su cálculo (Schreiber, 2007). En cuanto al estudio de la relación de las autocitas con el índice h de las revistas, hemos encontrado sólo un artículo en el área de aplicaciones informáticas de la base de datos Scopus, concluyendo que afecta al rango de las revistas (Ferrara y Romero, 2013). 
La constatación de la escasez de publicaciones sobre la visibilidad de las revistas iberoamericanas en Biblioteconomía/Bibliotecología y Documentación (ByD) a través de las citas recibidas en WoS (anteriormente ISI) ${ }^{1}$ y cómo afectan las autocitas al impacto de las publicaciones y al índice $\mathrm{h}$ de las revistas han sido las razones que nos han llevado a realizar este trabajo.

En línea con lo expuesto, el objeto general de este artículo es el estudio de la repercusión de los artículos de mayor impacto en WoS publicados en las dos revistas españolas de ByD listadas en el Journal Citation Reports (JCR), a saber: El Profesional de la Información (EPI) y Revista Española de Documentación Científica (REDOC).

En relación con dicho objeto general, los objetivos específicos de nuestro trabajo son:

- Conocer el impacto, medido en citas recibidas, según la procedencia de autores y revistas: iberoamericanas e internacionales.

- Contrastar si hay diferencia en el impacto de las dos revistas españolas listadas en el Journal Citation Reports (JCR): El Profesional de la Información (EPI) y Revista Española de Documentación Científica (REDOC).

- Determinar las revistas de las que procede el mayor número de citas.

- Conocer las tasas de autocitación, tanto de las revistas como de los autores.

- Determinar el impacto de las autocitas de las revistas en su índice h corregido y en el ranking de los artículos.

\section{Metodología}

Fuentes de datos. Se seleccionaron las revistas españolas de ByD listadas en el JCR: EPI y REDOC. El 1 de junio de 2017 se buscaron en la base de datos WoS los artículos publicados en dichas revistas.

Selección de los artículos. De cada revista se seleccionaron únicamente los artículos más citados: aquellos con un número de citas recibidas igual o superior al índice h. Para ello, se ordenaron los registros recuperados por el número de citas recibidas. El índice h de la revista EPI era de 14 y se seleccionaron 14 artículos. El índice h de REDOC era 11 y se seleccionaron 13 artículos que habían sido citados al menos 11 veces.

Extracción de datos. Se descargaron los datos bibliográficos de los artículos seleccionados y de los artículos citantes. De estos últimos, se examinaron

1 Respondiendo a dicho enfoque sólo hemos hallado un artículo (Herrero Solana y Liberatore, 2008), el cual no incluía la revista El Profesional de la Información, ya que en ese momento no estaba recogida en la base de datos citada. 
los autores y los títulos de las fuentes de publicación para calcular los siguientes indicadores:

- Autocitas de revista: número de citas recibidas por artículos publicados en la misma revista.

- Citas de revistas iberoamericanas: artículos publicados en español o portugués.

- Citas de revistas internacionales: artículos publicados en idioma distinto al español o portugués.

- Autocitas de autores: al menos un autor del artículo citante es autor del artículo citado, independientemente de su posición en el orden de firma en ambos artículos.

Para los propósitos de este trabajo, se ha utilizado el término "Iberoamérica" para referirnos a las revistas o autores de países de habla española o portuguesa de América, Portugal y España, y el término "Internacional" para revistas o autores cuyo idioma nativo es el inglés o cualquier otro que no sea español o portugués.

Además, se clasificaron las fuentes citantes en tres categorías: revistas incluidas en el JCR, revistas pertenecientes al Emerging Sources Citation Index (ESCI) y publicaciones procedentes del Conference Proceedings Citation Index.

Análisis de datos. El proceso y análisis estadístico de los datos se realizó con el programa SPSS versión 22. Para contrastar las diferencias entre las dos revistas, se aplicó el test de chi cuadrado o la t de Student según el nivel de medida de las variables. Se estudió también la correlación entre el índice h y el corregido sin autocitas.

\section{RESULTADOS Y DISCUSIÓN}

\section{Características generales de todos los artículos de las revistas}

El número de artículos publicados por EPI e indizados en WoS es casi tres veces más que en el caso de la revista REDOC (955 vs 346), proporción similar al número total de citas recibidas en ambas revistas (1 603 vs 613). A pesar de estas diferencias en las cifras absolutas, el porcentaje de artículos que no reciben ninguna cita es muy similar en ambas revistas ( $48.4 \%$ vs $49.4 \%$ ).

En cuanto a las citas recibidas de la propia revista, el porcentaje de autocitas en EPI es mayor que en REDOC (37.4 \% vs $32.1 \%)$, en consonancia 
con un menor porcentaje de artículos citantes procedentes de otras revistas $(70.8 \%$ vs $75.8 \%)$.

\begin{tabular}{|l|r|r|r|}
\cline { 2 - 4 } \multicolumn{1}{c|}{} & EPI & REDOC & \multicolumn{1}{c|}{$p^{a}$} \\
\hline $\mathrm{N}^{\circ}$ de artículos publicados & 955 & 346 & \\
\hline $\mathrm{N}^{\circ}$ artículos no citados & 462 & 171 & 0.739 \\
& $(48.4 \%)$ & $(49.4 \%)$ & \\
\hline $\mathrm{N}^{\circ}$ de artículos en que se cita & 1105 & 468 & \\
\hline $\mathrm{N}^{\circ}$ de artículos en que se cita sin citas propias & 783 & 355 & 0.043 \\
\hline Total de citas & $(70.8 \%)$ & $(75.8 \%)$ & \\
\hline Autocitas de revista & 1603 & 613 & \\
\hline Total sin autocitas revista & 600 & 197 & 0.020 \\
\hline Índice $\mathrm{h}$ & $(37.4 \%)$ & $(32.1 \%)$ & \\
\hline $\mathrm{N}^{\circ}$ de artículos que cumplen con el índice $\mathrm{h}$ & 1003 & 416 & \\
\hline
\end{tabular}

Tabla 1. Características generales de los artículos de EPIy REDOC en WoS a Comparación de proporciones: chi cuadrado

\section{Características de las citas de los artículos más citados}

El número total de citas recibidas por los artículos de EPI es muy superior a las recibidas por $R E D O C$, con un promedio de 18.5 citas recibidas por documento en EPI frente a las 13.1 citas por documento en REDOC $(\mathrm{p}=0.002)$.

$\mathrm{Al}$ analizar la procedencia de las citas, aunque no se observan diferencias estadísticamente significativas, destaca lo siguiente:

- El porcentaje de autocitas de revista es algo superior al $25 \%$ en ambas revistas, siendo un poco más alto en EPI que en REDOC, si bien esta diferencia es menor (1.6\%) que cuando se analizan todos los artículos publicados $(5.3 \%)$.

- El porcentaje de citas procedentes de revistas iberoamericanas es prácticamente el mismo en las dos revistas, el $42 \%$. Sin embargo, al distinguir entre nacionales (entendidas, dado el alcance del trabajo, como españolas) o no nacionales, EPI recibe un $2.5 \%$ más de citas nacionales mientras que REDOC recibe más citas de revistas de otros países iberoamericanos.

- En lo referente a las citas de revistas internacionales, ambas revistas obtienen porcentajes similares, casi un punto más en $R E D O C$ debido a las 
citas de los propios autores o de autores iberoamericanos, mientras que EPI recibe más citas de autores no iberoamericanos.

- El impacto de los artículos publicados en EPI y REDOC es principalmente en revistas en español o portugués, ya que dos de cada tres citas proceden de revistas iberoamericanas o son autocitas de revista. La repercusión en revistas en inglés es muy baja, sólo una de cada 10 es llevada a cabo por autores de países no iberoamericanos, y la mayoría de estas citas son a artículos publicados en inglés; así, en EPI, hay tres artículos publicados en inglés y escritos por autores no iberoamericanos que reciben 25 de las 30 citas, y en REDOC, 13 de las 17 citas las recibe un artículo publicado en inglés.

\begin{tabular}{|c|c|c|c|c|c|}
\cline { 2 - 5 } \multicolumn{1}{c|}{} & EPI & REDOC & \multicolumn{2}{c|}{$P^{b}$} & \multicolumn{2}{c}{} \\
\cline { 2 - 5 } \multicolumn{1}{c|}{} & $\mathrm{N}^{0}$ & $\%$ & $\mathrm{~N}^{0}$ & $\%$ & \\
\hline Citas de la propia revista & 71 & $27.3 \%$ & 44 & $25.7 \%$ & 0.717 \\
\hline Citas de revistas iberoamericanas & 110 & $42.3 \%$ & 73 & $42.7 \%$ & 0.937 \\
\hline Revistas nacionales & 69 & $26.5 \%$ & 41 & $24.0 \%$ & 0.551 \\
\hline Revistas no nacionales & 41 & $15.8 \%$ & 32 & $18.7 \%$ & 0.425 \\
\hline Citas de revistas internacionales & 79 & $30.4 \%$ & 54 & $31.6 \%$ & 0.793 \\
\hline Por propios autores & 22 & $8.5 \%$ & 16 & $9.4 \%$ & 0.748 \\
\hline Por autores iberoamericanos & 27 & $10.4 \%$ & 21 & $12.3 \%$ & 0.540 \\
\hline Por autores no iberoamericanos & 30 & $11.5 \%$ & 17 & $9.9 \%$ & 0.603 \\
\hline Total de citas & 260 & $100.0 \%$ & 171 & $100.0 \%$ & \\
\hline
\end{tabular}

Tabla 2. Características de citación de los artículos más citados de EPIy REDOC en WoS b Comparación de proporciones: chi cuadrado

\section{Revistas citantes}

Excluyendo las autocitas de las revistas, casi las tres cuartas partes de las citas proceden de revistas incluidas en el JCR y muy pocas son de actas de congresos. Existe un porcentaje importante de citas, el $22.2 \%$, que proceden de revistas que están en el Emerging Sources Citation Index (base de datos lanzada en 2015 para ampliar la cobertura geográfica a revistas de otros países de lengua no inglesa que están en proceso de evaluación antes de ser incluidas en el $J C R$ ), por lo que no tienen factores de impacto, pero sí se utilizan sus artículos en el conteo de citas; contribuyen, de esta manera, a engrosar los factores de impacto de las revistas JCR de aquellos países con menos impacto debido a la barrera del idioma en que publican. 
Al examinar las diferencias entre las dos revistas, en REDOC hay un porcentaje más alto de citas de revistas JCR, mientras que en EPI es mayor el de citas procedentes de revistas del Emerging Sources Citation Index. Así se desprende también de la información presentada en las Tablas 4 y 5 , en EPI hay cuatro revistas de fuentes emergentes en los primeros lugares, mientras que en $R E D O C$ sólo hay una revista proveniente de ESCI y con dos citas.

\begin{tabular}{|l|c|c|c|c|c|c|}
\cline { 2 - 7 } \multicolumn{1}{c|}{} & EPI & REDOC & Total & \multicolumn{3}{c|}{} \\
\cline { 2 - 7 } \multicolumn{1}{c|}{} & $\mathrm{N}^{0}$ & $\%$ & $\mathrm{~N}^{0}$ & $\%$ & $\mathrm{~N}^{0}$ & $\%$ \\
\hline $\begin{array}{l}\text { Revistas JCR sin autocitas } \\
\text { de revista }\end{array}$ & 133 & $70.4 \%$ & 98 & $77.2 \%$ & 231 & $73.1 \%$ \\
\hline $\begin{array}{l}\text { Emerging Sources Citation } \\
\text { Index }\end{array}$ & 48 & $25.4 \%$ & 22 & $17.3 \%$ & 70 & $22.2 \%$ \\
\hline $\begin{array}{l}\text { Conference Proceedings } \\
\text { Citation Index }\end{array}$ & 8 & $4.2 \%$ & 7 & $5.5 \%$ & 15 & $4.7 \%$ \\
\hline Total sin autocitas de revista & 189 & $100.0 \%$ & 127 & $100.0 \%$ & 316 & $100.0 \%$ \\
\hline
\end{tabular}

Tabla 3. Distribución de citas según la base de datos de revistas

El número de revistas que citan los artículos de EPI es muy superior al de REDOC (82 vs 59), algo lógico ya que el número de citas recibidas también es más alto. Según el idioma de publicación de la revista, en EPI es mayor el porcentaje de revistas internacionales $(52.4 \%)$, mientras que en REDOC es mayor en las revistas iberoamericanas $(61 \%)$.

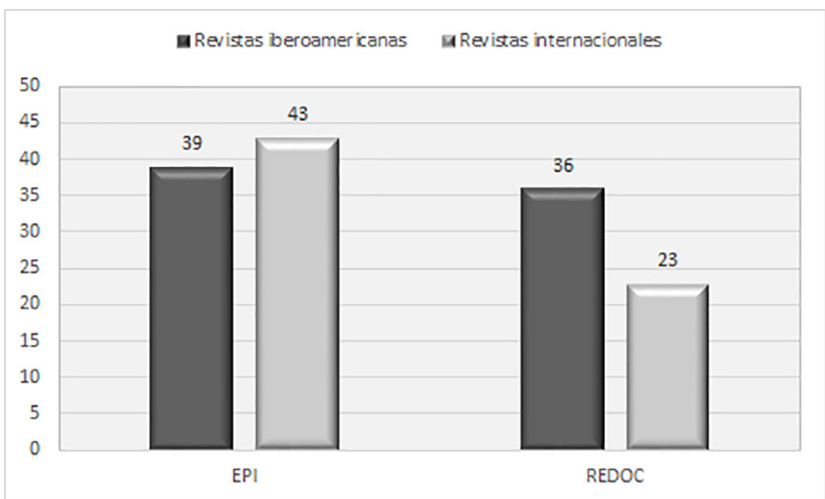

Figura 1. Distribución del número de revistas citantes según el idioma/país

Las revistas que más citan se presentan en las Tablas 4 y 5 . En ambos casos coinciden las tres revistas que más citas producen: la revista mexicana (Investigación Bibliotecológica), una española, que coincide con REDOC o EPI 
(según sea el caso) y una revista en inglés, Scientometrics. Cabe señalar que aunque el número de citas que suman estas tres revistas es más alto para EPI ( 56 citas) que para REDOC ( 33 citas), proceden del mismo número de artículos, 21 en ambos casos. También destaca que entre las revistas que más citan a EPI se encuentran más revistas procedentes del Emerging Sources Citation Index que en el caso de REDOC, y que las revistas internacionales más citantes están especializadas en temas de bibliometría y cienciometría.

\begin{tabular}{|c|c|c|c|}
\hline & \multirow{3}{*}{\begin{tabular}{r|}
$\mathbf{N}^{0}$ citas \\
21
\end{tabular}} & \multirow{3}{*}{$\frac{\mathrm{N}^{0} \text { artículos }}{8}$} \\
\hline & & & \\
\hline$J C R$ & Revista Española de Documentación Científica & & \\
\hline$J C R$ & Investigación Bibliotecológica & 19 & 9 \\
\hline$J C R$ & Scientometrics & 16 & 4 \\
\hline $\mathrm{ESCl}$ & Revista Latina de Comunicación Social & 7 & 4 \\
\hline$J C R$ & Journal of Informetrics & 7 & 3 \\
\hline$J C R$ & Comunicar & 6 & 4 \\
\hline$E S C l$ & Historia y Comunicación Social & 4 & 4 \\
\hline $\mathrm{ESCl}$ & $\begin{array}{l}\text { Revista Icono 14-Revista Científica de Comunicación } \\
\text { y Tecnologías }\end{array}$ & 5 & 3 \\
\hline$J C R$ & Transinformação & 3 & 3 \\
\hline $\mathrm{ESCl}$ & Observatorio & 3 & 3 \\
\hline$J C R$ & $\begin{array}{l}\text { Journal of the Association for Information Science and } \\
\text { Technology }\end{array}$ & 3 & 3 \\
\hline $\mathrm{CPCl}$ & $\begin{array}{l}\text { International Society of Scientometrics and Informetrics } \\
\text { Conference (ISSI) }\end{array}$ & 3 & 3 \\
\hline \multirow[t]{2}{*}{$J C R$} & Information Research & 3 & 3 \\
\hline & Total sin autocitas & 189 & 133 \\
\hline
\end{tabular}

Tabla 4. Revistas que más citan a EPI

* Las celdas sombreadas son revistas internacionales y las no sombreadas son revistas latinoamericanas

\begin{tabular}{|l|l|r|r|}
\cline { 3 - 4 } \multicolumn{2}{l|}{} & \multicolumn{1}{c|}{$\mathbf{N}^{\circ}$ citas } & \multicolumn{1}{c|}{$\mathbf{N}^{0}$ artículos } \\
\hline$J C R$ & Investigación Bibliotecológica & 14 & 9 \\
\hline$J C R$ & El Profesional de la Información & 10 & 5 \\
\hline$J C R$ & Scientometrics & 9 & 7 \\
\hline$C P C I$ & $\begin{array}{l}\text { International Society of Scientometrics and Informetrics } \\
\text { Conference (ISSI) }\end{array}$ & 7 & 3 \\
\hline$J C R$ & $\begin{array}{l}\text { Journal of the Association for Information Science } \\
\text { and Technology }\end{array}$ & 6 & 3 \\
\hline$J C R$ & Comunicar & & 2 \\
\hline
\end{tabular}




\begin{tabular}{|l|l|r|r|}
\hline$J C R$ & Journal of Informetrics & 5 & 3 \\
\hline$J C R$ & PLOS One & 3 & 3 \\
\hline$J C R$ & Transinformação & 2 & 2 \\
\hline$J C R$ & Revista Mexicana de Psicología & 2 & 2 \\
\hline$J C R$ & Revista de Psicología del Deporte & 2 & 2 \\
\hline$J C R$ & Online Information Review & 2 & 2 \\
\hline$J C R$ & Library Collections, Acquisition and Technical Services & 2 & 2 \\
\hline$J C R$ & Information Research & 2 & 2 \\
\hline ESCI & Aula Abierta & 2 & 2 \\
\hline$J C R$ & Adicciones & 127 & 2 \\
\hline
\end{tabular}

Tabla 5. Revistas que más citan a REDOC

* Las celdas sombreadas son revistas internacionales y las no sombreadas son revistas latinoamericanas

\section{Autocitación e indice b}

Si se tiene en cuenta de manera conjunta las autocitas de la revista y de los autores, se obtienen unas tasas de autocitación elevadas y muy parecidas en las dos revistas, $41.9 \%$ en EPI y $43.8 \%$ en REDOC. En ambos casos, la autocitación de revista es más alta que la que procede de los autores (Tabla 6).

$\mathrm{Al}$ analizar la distribución en las dos revistas, el porcentaje de autocitas de revista en $E P I$ es ligeramente mayor que en $R E D O C$, mientras que en ésta es mayor el porcentaje de autocitas de autores. La tasa de autocitación de autores, entre el $19.2 \%$ y el $24.6 \%$, es bastante más baja que el $47 \%$ obtenido por Jiménez Contreras, Delgado López Cózar y Ruiz Pérez (2006), lo que pensamos que puede ser debido a varios factores: en primer lugar, a que esta tasa se calculó con todos los trabajos publicados por autores españoles en WoS; en segundo lugar a que, tal y como observaron los propios autores, aquellos con más citas son los que presentan menor tasa de autocitación; en último lugar, al hecho de que el foco de nuestro estudio son los artículos más citados.

Al comparar la tasa de autocitación de la revista para todos los artículos incluidos en WoS (Tabla 1), se observa que en los artículos más citados es menor en ambos casos, 10.1 puntos menos en EPI y 6.4 puntos menos en $R E D O C$, lo que significa que los artículos menos citados en estas revistas aún tienen menos impacto real al presentar más citas que proceden de la propia revista. 


\begin{tabular}{|c|c|c|c|c|}
\cline { 2 - 3 } \multicolumn{1}{c|}{} & EPI & REDOC & \multicolumn{2}{c|}{} \\
\hline & $\mathrm{N}^{\circ}$ & $\%$ & $\mathrm{~N}^{0}$ & $\%$ \\
\hline Total de autocitas & 109 & $41.9 \%$ & 75 & $43.8 \%$ \\
\hline Autocitas de autores & 50 & $19.2 \%$ & 42 & $24.6 \%$ \\
\hline Autocitas de revista & 71 & $27.3 \%$ & 44 & $25.7 \%$ \\
\hline Autocitas autores en la revista & 12 & $4.6 \%$ & 11 & $6.4 \%$ \\
\hline Total de citas & 260 & $100.0 \%$ & 171 & $100.0 \%$ \\
\hline
\end{tabular}

Tabla 6. Tasa de autocitación en los artículos más citados de EPIy REDOC

En las Tablas 7 y 8 se presentan los artículos analizados con el número de citas totales y excluidas las autocitas, así como el rango obtenido una vez eliminadas las autocitas de revista, que son las únicas autocitas que se tienen en cuenta al calcular y corregir los indicadores de impacto de las revistas JCR. Estamos de acuerdo con Jiménez Contreras, Delgado López-Cózar y Ruiz Pérez (2006) cuando afirman que al establecer un escalafón deben excluirse las autocitas y, aunque en el contexto de su estudio se refieren a las autocitas de autor, creemos que debe extenderse a todo tipo de autocitas, de ahí que en las tablas presentemos la exclusión de las autocitas de autor y de revista.

$\mathrm{Al}$ excluir las autocitas de revista, en la revista EPI el índice h bajaría de 14 a 9, y en REDOC de 11 a 9, con lo que se igualarían en la base de datos. El descenso en el índice h depende no sólo de las autocitas sino también de que haya más artículos con un número de citas próximo al índice h; en este caso, se debe al mayor número de autocitas en EPI.

En cuanto al rango de los artículos, existen cambios importantes en algunos de ellos, aunque lógicamente, aquellos con mayor número de citas se ven menos afectados por la autocitación de la revista.

Sí se ha hallado en EPI una relación entre el número de autores firmantes y el número de autocitas de autores, de manera que a mayor número de autores mayor es la tasa de autocitación de autores (rho=0,606, p=0,022); sin embargo, en REDOC no se ha encontrado ningún tipo de relación (rho=-0,216; $\mathrm{p}=0,479)$. En la literatura también existen resultados contradictorios; así, Costas, Van Leeuwen y Bordons (2010) encontraron que el número de autocitas suele aumentar conforme mayor es el número de autores, pero Shah, Gul y Gaur (2015) no encontraron ningún tipo de relación en el campo de la ByD.

Aunque el número de artículos analizados en nuestra investigación no permite afirmar si existe algún tipo de patrón que pudiera explicar las tasas de autocitación, cabe destacar que los tres artículos publicados en EPI por 
autores internacionales $(\mathrm{R} 1=3, \mathrm{R} 1=6$ y $\mathrm{R} 1=8)$ tienen como máximo una autocita de la revista, si bien hay otros artículos de autores españoles que tampoco tienen autocitas de revista $(\mathrm{R} 1=5)$ o muy pocas $(\mathrm{R} 1=10, \mathrm{R} 1=14)$, coincidiendo en dos de ellos algunos autores. En REDOC no se observa ningún patrón, salvo la coincidencia de que dos artículos firmados por los mismos autores tienen tasas altas de autocitación $(\mathrm{R} 1=8, \mathrm{R} 1=10)$, coincidiendo también en EPI con el artículo con mayor tasa de autocitación de autores (R1=1).

\begin{tabular}{|l|c|c|c|c|c|c|}
\cline { 2 - 7 } \multicolumn{1}{l|}{} & A & B & C & D & R1 & R2 \\
\hline $\begin{array}{l}\text { Aleixandre-Benavent, R.; Valderrama-Zurian, } \\
\text { J.C.; Gonzalez-Alcaide, G. "Scientific journals } \\
\text { impact factor: limitations and alternative }\end{array}$ & 28 & 23 & 15 & 11 & 1 & 1 \\
indicators". 2007. & & & & & & \\
\hline $\begin{array}{l}\text { Casero-Ripolles, A. "Newspapers on the inter- } \\
\text { net: new business models in the convergence } \\
\text { era". 2010. }\end{array}$ & 27 & 16 & 24 & 14 & 2 & 5 \\
\hline $\begin{array}{l}\text { Leydesdorff, L.; Wagner, C.S.; Park, H-W; } \\
\text { Adams, J. "International collaboration in scien- } \\
\text { ce: the global map and the network". 2013. }\end{array}$ & $20^{\text {a }}$ & 20 & 16 & 16 & 3 & 2 \\
\hline $\begin{array}{l}\text { Rodriguez-Martinez, R.; Codina, L.; Pedraza-Ji- } \\
\text { menez, R. "Online journalism and web 2.0: A } \\
\text { model for analysis and report on results". 2010. }\end{array}$ & 21 & 12 & 19 & 10 & 4 & 9 \\
\hline $\begin{array}{l}\text { Torres-Salinas, D.; Jimenez-Contreras, E. } \\
\text { "Introduction and comparative study of the new } \\
\text { scientific journals citation indicators in Journal } \\
\text { Citation Reports and Scopus". 2010. }\end{array}$ & 20 & 20 & 20 & 20 & 5 & 3 \\
\hline $\begin{array}{l}\text { Jeremic, V.; Jovanovic-Milenkovic, M.; Radoji- } \\
\text { cic, Z.; Martic, M. "Excellence with leadership: } \\
\text { the Crown indicator of Scimago institutions } \\
\text { rankings Iber report". 2013. }\end{array}$ & 18 & 17 & 8 & 8 & 6 & 4 \\
\hline $\begin{array}{l}\text { Guallar, J.; Abadal, E. "Evaluation of digital } \\
\text { press archives: indicators and examples of good } \\
\text { practice". 2009. }\end{array}$ & 18 & 9 & 13 & 6 & 7 & 10 \\
\hline $\begin{array}{l}\text { Leydesdorff, L. "World shares of publications of } \\
\text { the USA, EU-27, and China compared and pre- } \\
\text { dicted using the new Web of Science interface } \\
\text { versus Scopus". 2012. }\end{array}$ & 16 & 15 & 15 & 14 & 8 & 6 \\
\hline Diaz-Noci, J. "Trends in internet media". 2010. & 16 & 6 & 16 & 6 & 9 & 14 \\
\hline $\begin{array}{l}\text { Torres-Salinas, D.; Bordons, M.; Gimenez-To- } \\
\text { ledo, E.; Delgado-Lopez-Cozar, E.; Jime- } \\
\text { nez-Contreras, E.; Sanz-Casado, E. "Integrated } \\
\text { scientific journals classification (CIRC): A } \\
\text { proposal for categorization of journals in social } \\
\text { sciences and humanities". 2010. }\end{array}$ & 16 & 14 & 11 & 10 & 10 & 7 \\
\hline
\end{tabular}




\begin{tabular}{|l|c|c|c|c|c|c|}
\hline $\begin{array}{l}\text { Peset, F.; Ferrer-Sapena, A.; Subirats-Coll, I. } \\
\text { "Linked open data and Open data. Its impact in } \\
\text { the field of libraries and information science". } \\
\text { 2011. }\end{array}$ & 15 & 7 & 14 & 7 & 11 & 12 \\
\hline $\begin{array}{l}\text { Guallar, J.; Rovira, C.; Ruiz, S. "Multimedia } \\
\text { in digital media. Multimedia elements and } \\
\text { retrieval systems in the leading Spanish online } \\
\text { newspapers". 2010. }\end{array}$ & 15 & 7 & 12 & 7 & 12 & 13 \\
\hline $\begin{array}{l}\text { Cabezas-Clavijo, A.; Torres-Salinas, D.; } \\
\text { Delgado-Lopez-Cozar, E. "Science 2.0: Tools } \\
\text { catalogue and consequences for scientific } \\
\text { activity". 2009. }\end{array}$ & 15 & 9 & 12 & 8 & 13 & 11 \\
\hline $\begin{array}{l}\text { Costas, R.; Bordons, M. "A critical view of the } \\
\text { h-index: observations based on a practical } \\
\text { application". 2007. }\end{array}$ & 15 & 14 & 15 & 14 & 14 & 8 \\
\hline \\
A: No de citas en la base de datos, B: No de citas sin autocitas de la revista; C: No de citas \\
sin autocitas de los autores; D: No de citas sin autocitas; R1: Rango del artículo según \\
número de citas; R2: rango del artículo sin autocitas de revista \\
a Corresponde al número real de citas que se pueden descargar del WoS, \\
aunque en el artículo aparece con 1 cita más
\end{tabular}

Tabla 7. Rango y número de citas sin autocitas de los artículos de EPI

\begin{tabular}{|c|c|c|c|c|c|c|}
\hline & $A$ & B & C & D & R1 & R2 \\
\hline $\begin{array}{l}\text { Torres-Salinas, D.; Robinson-Garcia, N.; } \\
\text { Jimenez-Contreras, E.; Delgado Lopez-Cozar, } \\
\text { E. "Towards a Book Publishers Citation Reports. } \\
\text { First approach using the Book Citation Index”. } \\
2012 .\end{array}$ & 25 & 24 & 18 & 18 & 1 & 1 \\
\hline $\begin{array}{l}\text { Bordons, M.; Sancho, R.; Morillo, F.; Gómez, I. } \\
\text { "Scientific activity profile of Spanish universities } \\
\text { in four thematic areas: a multifactor analysis". } \\
2010 .\end{array}$ & 17 & 9 & 15 & 9 & 2 & 6 \\
\hline $\begin{array}{l}\text { Rodriguez-Martinez, R.; Codina, L.; Pedraza-Ji- } \\
\text { menez, R. "Indicators to evaluate the quality of } \\
\text { online journalism web sites: an analysis of Web } \\
2.0 \text { interaction and adoption". } 2012 \text {. }\end{array}$ & 13 & 10 & 11 & 8 & 3 & 3 \\
\hline $\begin{array}{l}\text { De Filippo, D.; Sanz-Casado, E.; Urbano Salido, } \\
\text { C.; Ardanuy, J.; Gomez-Caridad, I. "The role } \\
\text { of institutional databases in the analysis of } \\
\text { universities' scientific activity”. } 2011 .\end{array}$ & 13 & 9 & 12 & 9 & 4 & 7 \\
\hline $\begin{array}{l}\text { Dorta-Gonzalez, P.; Dorta-Gonzalez, M.I. } \\
\text { "Bibliometric indicator based on the h-index". } \\
2010 .\end{array}$ & 13 & 12 & 6 & 6 & 5 & 2 \\
\hline
\end{tabular}




\begin{tabular}{|c|c|c|c|c|c|c|}
\hline $\begin{array}{l}\text { Fernández Quijada, F. "The profile of Spanish } \\
\text { journals in the field of communication (2007- } \\
\text { 2008)". } 2010 .\end{array}$ & 12 & 10 & 5 & 4 & 6 & 4 \\
\hline $\begin{array}{l}\text { Ortega, J.L.; Aguillo, I. "Structural analysis of } \\
\text { the Iberoamerican academic web". } 2009 .\end{array}$ & 12 & 10 & 11 & 9 & 7 & 5 \\
\hline $\begin{array}{l}\text { Gonzalez Alcaide, G.; Castellano Gomez, M.; } \\
\text { Valderrama Zurian, J.C.; Aleixandre Benavent, } \\
\text { R. "Scientific literature by Spanish authors on } \\
\text { the analysis of citations and impact factor in } \\
\text { Biomedicine (1981-2005)". 2008. }\end{array}$ & $11^{\mathrm{a}}$ & 5 & 6 & 3 & 8 & 13 \\
\hline $\begin{array}{l}\text { De Filippo, D.; Morillo, F.; Teresa Fernandez, M. } \\
\text { "Indicators of scientific collaboration between } \\
\text { CSIC and Latin America through international } \\
\text { databases". } 2008 .\end{array}$ & 12 & 8 & 8 & 6 & 9 & 10 \\
\hline $\begin{array}{l}\text { Gonzalez Alcaide, G.; Valderrama Zurian, J.C.; } \\
\text { Aleixandre Benavent, R. "Analysis of the inter- } \\
\text { nationalization process of Spanish research in } \\
\text { science and technology (1980-2007)". } 2012 .\end{array}$ & $10^{\mathrm{a}}$ & 6 & 6 & 4 & 10 & 11 \\
\hline $\begin{array}{l}\text { Jimenez-Contreras, E.; Robinson-Garcia, N.; } \\
\text { Cabezas-Clavijo, A. "Productivity and impact } \\
\text { of Spanish researchers: reference thresholds } \\
\text { within scientific areas". } 2011 .\end{array}$ & 11 & 6 & 10 & 5 & 11 & 12 \\
\hline $\begin{array}{l}\text { Torres-Salinas, D.; Muñoz-Muñoz, A.M.; } \\
\text { Jimenez-Contreras, E. "Bibliometric analysis of } \\
\text { the situation of female researchers in the fields } \\
\text { of social sciences and law in Spain". } 2011 .\end{array}$ & 11 & 9 & 11 & 9 & 12 & 8 \\
\hline $\begin{array}{l}\text { Carlos Sierra, J; Buela-Casal, G.; Bermudez } \\
\text { Sanchez, M.P.; Santos-Iglesias, P. "Opinion } \\
\text { of Associate Professors and Full Professors } \\
\text { regarding the criteria and standards for the } \\
\text { accreditation of Spanish university teachers". } \\
2009\end{array}$ & 11 & 9 & 10 & 8 & 13 & 9 \\
\hline \multicolumn{7}{|c|}{ 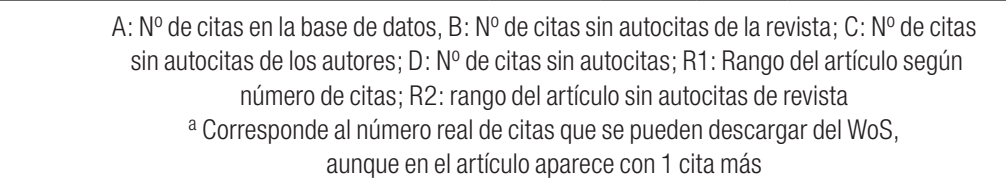 } \\
\hline
\end{tabular}

Tabla 8. Rango y número de citas sin autocitas de los artículos de REDOC

\section{CONCLUSIONES Y RECOMENDACIONES}

De los resultados obtenidos, y atendiendo a los objetivos propuestos, se derivan las siguientes conclusiones y recomendaciones: 
La repercusión de los artículos más citados publicados en las revistas españolas EPI y REDOC se produce principalmente en la comunidad científica iberoamericana, lo que significa que su impacto internacional es muy bajo, ya que sólo una de cada 10 citas proceden de autores no iberoamericanos en revistas internacionales, y la mayoría de ellas las reciben artículos publicados en inglés. Por lo tanto, los artículos analizados publicados en las revistas españolas seleccionadas tienen muy bajo impacto internacional y son citados por autores que publican en revistas que utilizan este idioma como vehículo de comunicación.

El impacto de los artículos de EPI y REDOC en revistas iberoamericanas e internacionales es muy similar. En EPI la revista con mayor número de citas es REDOC, seguida de la revista mexicana Investigación Bibliotecológica y de una revista internacional publicada en inglés, Scientometrics. En REDOC, las tres revistas más citantes son las mismas, salvo que el orden es primero para Investigación Bibliotecológica y EPI en segunda posición.

Existe una tasa muy alta de autocitación (cerca del $40 \%$ ), por lo que la repercusión externa de los artículos es baja y su impacto (número de citas) depende en cierta medida de los propios autores y de la revista.

La eliminación de las autocitas de revista afecta al índice h y al orden de los artículos. Al igual que se calculan en el JCR los indicadores de impacto corregidos por las autocitas, pensamos que sería recomendable que se publicara también el índice $\mathrm{h}$ sin autocitas en todas las bases de datos, con la finalidad de dar una aproximación más real y objetiva de su impacto en la comunidad científica.

Hay ocasiones en que la autocitación está justificada, pero otras veces se detecta una tasa excesivamente alta de autocitas o están injustificadas, al no tener relación con trabajos previos. Las autocitas de autores proporcionan información valiosa sobre el proceso de comunicación científica, pero si se desea evaluar el impacto de un trabajo y/o autor, las citas externas son más relevantes, ya que reflejan la repercusión en la comunidad científica y, por consiguiente, pensamos que deberían ser eliminadas las autocitas.

Sería deseable averiguar si la autocitación y el impacto de los estudios publicados en revistas iberoamericanas en otras áreas de conocimiento siguen los mismos patrones que los obtenidos en el campo de la $\mathrm{ByD}$ y si su repercusión se centra casi exclusivamente entre la comunidad científica de la misma región. 


\section{REFERENCIAS}

Aksnes, Dag W. 2003. "A macro-study of self-citation”. Scientometrics 56 (2): 235 246. doi: $10.1023 / \mathrm{A}: 1021919228368$

Bartneck, C. y S. Kokkelmans. 2011. "Detecting h-index manipulation through self-citation analysis". Scientometrics 87 (1): 85-98. doi: 10.1007/s11192-010-0306-5

Bordons, María, M. T. Fernández e Isabel Gómez. 2002. "Advantages and limitations in the use of impact factor measures for the assessment of research performance in a peripheral country”. Scientometrics 53 (2): 195-206. doi: 10.1023/A:1014800407876

Braun, Tibor, Wolfgang Glänzel y András Schubert. 2006. "A Hirsch-type index for journals”. Scientometrics 69 (1): 169-173. doi: 10.1007/s11192-006-0147-4

Campanario, Juan Miguel. 2010. "Self-citations that contribute to the journal impact factor: An investment-benefit-yield analysis". Journal of the American Society for Information Science and Technology 61 (12): 2575-2580. doi: 10.1002/asi.21439

Costas, Rodrigo, Thed N. van Leeuwen y María Bordons. 2010. "Self-citation at the meso and individual levels: effects of different calculation methods". Scientometrics 82 (3): 517-537. doi: 10.1007/s11192-010-0187-7

Chorus, Caspar y Ludo Waltman. 2016. "A large-scale analysis of impact factor biased journal self-citations”. PLOS One 11 (8): e0161021. doi:10.1371/journal.pone.0161021

De Granda Orive, José Ignacio, Adolfo Alonso Arroyo y Rafael Aleixandre Benavent. 2014. “Autocitación: ¿debemos penalizarla?”. Archivos de Bronconeumología 50 (10): 458. doi: 10.1016/j.arbres.2013.10.008

Egghe, Leo y Ronald Rousseau. 1990. Introduction to Informetrics: quantitative methods in library, documentation and information science. Amsterdam: Elsevier Science Publishers.

Espinet Rubio, Pablo. 2006. "Perversiones, paradojas y traumas en la evaluación científica”. Anales de Química 102 (4): 60-63.

Ferrara, Emilio y Alfonso E. Romero. 2013. "Scientific impact evaluation and the effect of self-citations: mitigating the bias by discounting h-index". Journal of the American Society for Information Science and Technology 64 (11): 2332-2339. doi: 10.1002/asi.22976

Finardi, Ugo. 2013. "Correlation between journal impact factor and citation performance: An experimental study". Journal of Informetrics 7 (2): 357-370. doi: 10.1016/j.joi.2012.12.004

Foley, Jennifer A. y Sergio Della Sala. 2010. "The impact of self-citation”. Cortex 46 (6): 802-810. doi: 10.1016/j.cortex.2010.01.004

Frandsen, Tove Faber. 2007. "Journal self-citations - analyzing the JIF mechanism". Journal of Informetrics 1 (1): 47-58. doi: 10.1016/j.joi.2006.09.002

Gianoli, Ernesto y Marco A. Molina-Montenegro. 2009. "Insights into the relationship between the h-index and self-citation". Journal of the American Society for Information Science and Technology 60 (6): 1283-1285. doi: 10.1002/asi.21042

Gorbea-Portal, Salvador y Carlos A. Suárez-Balseiro. 2007. "Análisis de la influencia y el impacto entre revistas periféricas no incluidas en el Science Citation Index". Revista Interamericana de Bibliotecología 30 (2): 47-70.

Herrero Solana, Víctor y Gustavo Liberatore. 2008. "Visibilidad internacional de las revistas iberoamericanas de Bibliotecología y Documentación”. Revista Española de Documentación Cientifica 31 (2): 230-239. 
Hirsch, J. E. 2005. "An index to quantify an individual's scientific research output". Proceedings of the National Academy of Sciences of the United States of America 102 (46): 16569-16572. doi:10.1073/pnas.0507655102

Hyland, Ken. 2003. "Self-citation and self-reference: Credibility and promotion in academic publication". Journal of the American Society for Information Science and Technology 54 (3): 251-259. doi: 10.1002/asi.10204

Ioannidis, John P.A. 2015. "A generalized view of self-citation: Direct, co-author, collaborative, and coercive induced self-citation". Journal of the Psychosomatic Research 78 (1): 7-11. doi: 10.1016/j.jpsychores.2014.11.008

Jiménez Contreras, Evaristo, Emilio Delgado López-Cózar y Rafael Ruiz Pérez. 2006. "Producción española en biblioteconomía y documentación con visibilidad internacional a través del Web of Science (1995-2004)”. El Profesional de la Información 15 (5): 373-383.

Krauss, Jochen. 2007. "Journal self-citattion rates in ecological sciences". Scientometrics 73 (1): 79-89. doi: 10.1007/s11192-007-1727-7

McVeigh, M. E. 2002. Journal self-citation in the Journal Citation Reports - Science Edition (2002). Fecha de consulta: 13 de septiembre de 2017. http://wokinfo. com/essays/journal-self-citation-jcr/

Opthof, T. 2013. "Inflation of impact factors by journal self-citation in cardiovascular science". Netherlands Heart Journal 21 (4): 163-165. doi: 10.1007/s12471-013-0384-0

Salvador Oliván, José Antonio y Carmen Agustín Lacruz. 2015. "Correlación entre indicadores bibliométricos en revistas de Web of Science y Scopus". Revista General de Información y Documentación 25 (2): 341-359. http://dx.doi.org/10.5209/ rev_RGID.2015.v25.n2.51241

Schekman, Randy. 2013. "How journals like Nature, Cell ad Science are damaging science". The Guardian. Fecha de consulta: 12 de septiembre de 2017. https:// www.theguardian.com/commentisfree/2013/dec/09/how-journals-nature-science-cell-damage-science

Schreiber, M. 2007. "Self-citation corrections for the Hirsch index". Europhysics Letters 78 (3): 30002. doi: 10.1209/0295-5075/78/30002

Shah, Tariq Ahmad, Sumeer Gul y Ramesh C. Gaur. 2015. "Authors self-citation behavior in the field of Library and Information Science". Aslib Journal of Information Management 67(4): 48-468. doi: 10.1108/AJIM-10-2014-0134

Vanclay, Jerome K. 2012. "Impact factor: outdated artefact or stepping-stone to journal certification?”. Scientometrics 92 (2): 211-238. doi: 10.1007/s11192-011-0561-0

Wilhite, Allen W. y Eric A. Fong. 2012. "Coercitive citation in academic publishing”. Science 335 (6068): 542-543. doi: 10.1126/science.1212540

Para citar este texto:

Salvador-Oliván, José Antonio, Gonzalo Marco-Cuenca y Rosario Arquero-Avilés. 2018. "Impacto de las revistas españolas de Biblioteconomía y Documentación y repercusión de las autocitas en su índice h”. Investigación Bibliotecológica: archivonomía, bibliotecología e información 32 (77): 13-30. 